\title{
MicroRNA-34a affects chondrocyte apoptosis and proliferation by targeting the SIRT1/p53 signaling pathway during the pathogenesis of osteoarthritis
}

\author{
SHIJU YAN*, MENG WANG* ${ }^{*}$ JIAN ZHAO, HONGTAO ZHANG, CHENGPEI ZHOU, LEI JIN, \\ YINGLONG ZHANG, XIUCHUN QIU, BAOAN MA and QINGYU FAN
}

Department of Orthopedics, Tangdu Hospital, Fourth Military Medical University, Xi'an, Shaanxi 710038, P.R. China

Received July 22, 2015; Accepted May 16, 2016

DOI: $10.3892 /$ ijmm.2016.2618

\begin{abstract}
Osteoarthritis (OA) is the most prevalent degenerative joint disease with multifactorial etiology caused by risk factors such as ageing, obesity and trauma. Previously, it was reported that the inhibition of microRNA-34a (miR-34a) may reduce rat chondrocyte apoptosis induced by IL- $1 \beta$, whereas the molecular mechanism and the role of miR-34a in human chondrocyte as well as in OA progression remains to be determined. In the current study, using MTT, luciferase reporter assays and western blot analysis we identified that miR-34a was upregulated while silent information regulator 1 (SIRT1) was inhibited in chondrocytes from $12 \mathrm{OA}$ patients compared with healthy chondrocytes from 10 trauma amputees. Overexpression of miR-34a promoted apoptosis and inhibited cell proliferation in human chondrocytes. Transfection with miR-34a mimic inhibited SIRT1 expression, which attenuated the deacetylation of $\mathrm{p} 53$, leading to the upregulation of Bax and downregulation of Bcl-2. Furthermore, results from the western blot analysis and luciferase reporter assay demonstrated that SIRT1 was directly regulated by miR-34a in human chondrocytes. A rat model of OA was induced through anterior cruciate ligament transection and medial meniscus resection (ACLT+MMx). The results showed that the intra-articular injection of lentiviral vector encoding anti-miR-34a sequence effectively ameliorated the progression of OA. The results suggest that miR-34a has a crucial role in the pathogenesis of OA through direct regulation of the SIRT1/p53 signaling pathway and serves as a potential therapeutic target of OA.
\end{abstract}

Correspondence to: Professor Baoan Ma or Professor Qingyu Fan, Department of Orthopedics, Tangdu Hospital, Fourth Military Medical University, 1 Xinsi Road, Xi'an, Shaanxi 710038, P.R. China E-mail: gukemba@fmmu.edu.cn

E-mail: bonetm@fmmu.edu.cn

${ }^{*}$ Contributed equally

Key words: microRNA-34a, silent information regulator 1, p53, apoptosis, osteoarthritis, chondrocyte, lentivirus

\section{Introduction}

Osteoarthritis (OA) is a widely prevalent age-associated joint disorder characterized by the progressive destruction of cartilage and is responsible for the deterioration of life quality and economic burden in the elderly population worldwide $(1,2)$. It is known that OA involves all structures in the affected joints, resulting in joint instability, pain, and loss of functions $(3,4)$. Chondrocyte is the only cell type present in articular cartilage and plays a critical role in maintaining the dynamic equilibrium between anabolism and catabolism of the extracellular matrix (ECM) under physiological circumstances, which is crucial for joint function (5).

OA has various underlying pathogenic mechanisms that are not fully understood. Apoptosis, or programmed cell death, has been connected with OA $(6,7)$. It is reported that OA cartilage has a higher proportion of apoptotic chondrocytes than normal tissue and apoptotic cells are located in the superficial and middle zones (6). Previous findings suggested that chondrocyte apoptosis plays a key role in cartilage degradation and revealed a significant correlation between apoptosis and the severity of OA progression, indicating that inhibition of chondrocyte apoptosis may be an important strategy for OA treatment (7).

MicroRNAs consist of a class of single-stranded non-coding RNAs of 18-24 nucleotides that are evolutionarily conserved (8). MicroRNAs can regulate gene expression by binding to the 3'-untranslated region (3'-UTR) of their target mRNAs, leading to post-transcriptional repression or mRNA cleavage $(9,10)$. MicroRNAs are involved in a number of physiological functions and disease processes, and a growing body of evidence has elucidated the physiologic and pathogenetic role of microRNAs in the maintenance of joint homeostasis and the development of OA, such as inflammation, cellular communication and cell death $(11,12)$. MicroRNA-34a (miR-34a) is important as a tumor suppressor gene in downregulating its target genes. miR-34a is also involved in p53-induced cell cycle arrest, cell senescence, apoptosis and other biological behaviors $(13,14)$.

Silent information regulator 1 (SIRT1) is a conserved nicotinamide adenine dinucleotide $\left(\mathrm{NAD}^{+}\right)$-dependent deacetylase that plays a crucial role in apoptosis, cell senescence, inflammation and tumorigenesis by deacetylating important transcriptional factors, including p53, FOXO and p65 (15-17). 
In a previous study, silencing of miR-34a effectively reduced rat chondrocyte apoptosis and the downregulation of Col2a1 induced by IL-1 $\beta$ (18). However, the underlying mechanism and the role of miR-34a in the progression of OA remain to be determined. Additionally, the overexpression of SIRT1 protects chondrocytes from osteoarthritic change induced by IL-1 $\beta$ (19). miR-34a is reported to induce apoptosis in human colon cancer cells by inhibiting SIRT1 (20). Thus, in the current study, we hypothesized whether miR-34a was able to promote apoptosis in human chondrocyte by targeting the SIRT1/p53 signaling pathway.

First, we found an increased miR-34a expression and a decreased level of SIRT1 in human OA chondrocytes. Second, the overexpression of miR-34a induced apoptosis and inhibited cell proliferation in human chondrocytes. In addition, miR-34a repressed the expression of SIRT1 by binding 3'-UTR of SIRT1 mRNA, which inhibited the deacetylation of p53, leading to the regulation of downstream genes Bax and Bcl-2. Intra-articular injection of lentivirus encoding anti-miR-34a oligonucleotide ameliorated the progression of OA induced by surgery in rats. These findings indicated that miR-34a is a promising gene therapeutic target in OA.

\section{Materials and methods}

Patients. The protocol was approved by the Institutional Review Board of Tangdu Hospital (approval ID no. TDLL-2013009) (Shaanxi, China) and written informed consent was obtained from all the participating patients. OA articular cartilage samples were collected from the femoral condyles and tibial plateaus of 12 patients with primary OA undergoing total knee arthroplasty (TKA) (mean age $\pm \mathrm{SD}, 63.1 \pm 9.1$ years) at the Department of Orthopedics of Tangdu Hospital. Healthy cartilage samples were obtained from 10 trauma patients with no history of OA or other joint diseases $(46.8 \pm 12.7$ years $)$.

Isolation and primary culture of human articular chondrocytes. To isolate primary human chondrocytes, cartilage specimens were dissected into small sections and subjected to sequential digestion with $0.1 \%$ trypsin (Invitrogen, Carlsbad, CA, USA) for $30 \mathrm{~min}$ and then with $0.2 \%$ collagenase Type II (Millipore Corp., Billerica, MA, USA) in Dulbecco's modified Eagle's medium(DMEM) (Gibco) for $10 \mathrm{~h}$ at $37^{\circ} \mathrm{C}$. Isolated chondrocytes were filtered through $100-\mu \mathrm{m}$ nylon filters. The cells were seeded in culture flasks in DMEM/F12 medium (Gibco) supplemented with $10 \%$ fetal bovine serum (FBS) (HyClone, Thermo Fisher Scientific, Waltham, MA, USA), 100 U/ml penicillin and $100 \mu \mathrm{g} / \mathrm{ml}$ streptomycin (Gibco), and incubated at $37^{\circ} \mathrm{C}$ in a humidified atmosphere with $5 \% \mathrm{CO}_{2}$.

Primary chondrocytes were used to compare gene expression levels in normal and OA chondrocytes. The cells from passages 1 to 2 were used for the subsequent experiments.

RNA extraction and measurement of $m R N A$ and microRNA expression. Total RNA was extracted from primary chondrocytes using TRIzol reagent (Invitrogen) according to the manufacturer's instructions. RNA was reverse-transcribed to generate first-strand complementary DNA (cDNA) using high-capacity cDNA reverse transcription kits (Applied Biosystems Life Technologies, Foster City, CA, USA).
Real-time PCR was performed with the Power SYBR-Green PCR Master Mix and 7900 HT Fast Real-Time PCR system (both from Applied Biosystems, Carlsbad, CA, USA). The data were given as a threshold cycle $(\mathrm{Ct})$. The levels of mRNA were normalized to glyceraldehyde 3-phosphate dehydrogenase (GAPDH) mRNA controls using the comparative $2^{-\Delta \Delta \mathrm{Ct}}$ method. The specific primers used for different mRNAs were: for human SIRT1, 5'-TGGCAAAGGAGCAGATTAGT AGG-3' (forward) and 5'-CTGCCACAAGAACTAGAGG ATAAGA-3' (reverse); for human GAPDH, 5'-ATTCCACCC ATGGCAAATTC-3' (forward) and 5'-TGGGATTTCCAT TGATGACAAG-3' (reverse).

To evaluate the miR-34a expression levels, total RNA was reverse transcribed into cDNA using the TaqMan MicroRNA Reverse Transcription kit and TaqMan MicroRNA assays (Applied Biosystems) according to the manufacturer's instructions. The small nuclear RNA U6 (RNU6) was used as an endogenous control for miRNA detection.

Transfection of microRNA. miR-34a mimic, miR-34a inhibitor and non-specific negative control (NC) were synthesized and purified by Guangzhou RiboBio Co., Ltd. (Guangzhou, China). There was a fluorescent FAM label linked with the 5' terminal of miR-34a mimic, which was used for transfection validation. When the chondrocytes were grown to $80 \%$ confluence, miR-34a mimic, miR-34a inhibitor or non-specific negative oligonucleotides were transfected at a working concentration of $100 \mathrm{nmol} / \mathrm{l}$ using Lipofectamine 2000 (Invitrogen) according to the manufacturer's instructions. Twenty-four hours after incubation, the transfection efficiency was observed using a fluorescence microscope (Olympus Co., Ltd., Beijing, China) and miR-34a expression level was evaluated by quantitative PCR.

In vitro cell proliferation assay. Cell proliferation was measured with 3-(4,5-dimethylthiazol-2-yl)-2,5-diphenyltetrazolium bromide (MTT) assay. Chondrocytes were seeded in 96-well plates at a density of $5 \times 10^{3}$ cells/well containing $100 \mu \mathrm{l}$ of culture medium. The cells were transfected with $100 \mathrm{nM}$ miR-34a mimic, miR-34a inhibitor or non-specific NC for $6 \mathrm{~h}$. After transfection, $20 \mu \mathrm{l}$ of MTT $(5 \mathrm{mg} / \mathrm{ml})$ (Sigma-Aldrich, St. Louis, MO, USA) was added every $24 \mathrm{~h}$, followed by incubation for another $4 \mathrm{~h}$ at $37^{\circ} \mathrm{C}$. The medium was removed, and $100 \mu \mathrm{l}$ of dimethyl sulfoxide was added to each well to dissolve the formazan. The optical density (OD) was evaluated by measuring the absorbance at the wavelength of $490 \mathrm{~nm}$, with a reference wavelength of $630 \mathrm{~nm}$, using a microplate reader (Multiskan Ascent 354; Thermo Labsystems, Vantaa, Finland).

Apoptosis assay. The apoptotic rate of chondrocytes was detected and quantified by flow cytometry. Briefly, chondrocytes were transfected with $100 \mathrm{nM}$ miR-34a mimic, miR-34a inhibitor or NC. Forty-eight hours after transfection, $1 \times 10^{5}$-treated cells of each group were collected, washed and incubated with Annexin V-FITC and propidium iodide (PI) (both from Millipore Corp.) for $15 \mathrm{~min}$ at a room temperature of $20^{\circ} \mathrm{C}$ in the dark. The cells were analyzed using a fluorescenceactivated cell sorting (FACS) flow cytometer (BD Biosciences, San Diego, CA, USA). 
Western blot analysis. Total proteins were extracted from the cells using RIPA buffer (Beyotime Institute of Biotechnology, Shanghai, China) with enzyme inhibitor cocktail (Complete; Roche, Basel, Switzerland). After being lysed on ice for 30 $\mathrm{min}$, the lysate was centrifuged at $13,000 \mathrm{x} \mathrm{g}$ for $20 \mathrm{~min}$, and the supernatant was collected for experiments. For the western blot analysis, $25 \mu \mathrm{g}$ of extracts were separated using $10 \%$ SDS-polyacrylamide gel electrophoresis, transferred to a polyvinylidene difluoride membrane (Millipore Corp., Bedford, MA, USA) and incubated with anti-SIRT1 (mouse monoclonal antibody; 1:1,000; Cat. no. 8469), acetylated-p53 (Lys382; rabbit polyclonal antibody; 1:1,000; Cat. no. 2525), p53 (rabbit monoclonal antibody; 1:1,000, Cat. no. 2527), Bax (rabbit monoclonal antibody; 1:1,000; Cat. no. 5023), Bcl-2 (rabbit monoclonal antibody; 1:1,000; Cat. no. 4223) (Cell Signaling Technology, Inc., Danvers, MA, USA) and anti-GAPDH (mouse monoclonal antibody; 1:500; Cat. no. sc-365062; Santa Cruz Biotechnology, Inc., Santa Cruz, CA, USA) antibodies for $24 \mathrm{~h}$ at $4^{\circ} \mathrm{C}$. GAPDH was used as internal control. The blots were developed using a chemiluminescent substrate kit (Pierce Biotechnology, Inc., Rockford, IL, USA). Intensity of the bands was detected and analyzed using Quantity One analyzing system (Bio-Rad Laboratories, Inc., Hercules, CA, USA).

Luciferase reporter assay. Total RNA (1 $\mu \mathrm{g})$ from human chondrocytes was reverse-transcribed into cDNA, and the SIRT1 3'-UTR was amplified using the primers: 5'-ATAGGC CGGCATAGACGCGTTGTAATAATTGTGCAGGTAC AGG-3' (forward) and 5'-AAAGATCCTTTATTAAGCTTA AGTTAACAGAAAAAAGTCAAATGAC-3' (reverse). The 3'-UTR of SIRT1 (1,796 bp) containing the miR-34a binding sites was cloned into the HindIII and MluI sites of the pMIR-Report Luciferase vector (Ambion, Austin, TX, USA) downstream of the firefly luciferase gene to develop the wild-type 3'-UTR luciferase reporter vector. The mutant 3'-UTR luciferase reporter vector was generated by site-directed mutagenesis using the QuikChange Site-Directed Mutagenesis kit (Stratagene, La Jolla, CA, USA). Human chondrocytes were plated in 24-well plates. The cells at $70-80 \%$ confluence were co-transfected with wild-type or mutant-type 3'-UTR-Luc reporter vector plus miR-34a mimic with Lipofectamine 2000 ( Invitrogen). The pRL-CMV Renilla luciferase vector (Promega Corp., Madison, WI, USA) was used to normalize cell numbers and transfection efficiency. After an additional $48 \mathrm{~h}$, the luciferase activity was measured using the dual luciferase assay (Promega Corp.) according to the manufacturer's instructions.

Construction of lentivirus vector. Recombinant lentivirus vector encoding antisense miR-34a (anti-miR-34a) or negative control (miR-NC) were constructed and purchased from GeneChem Co., Ltd. (Shanghai, China). Briefly, the oligonucleotides of antisense miR-34a inhibitor and $\mathrm{NC}$ were cloned into the lentivirus expression vector of hU6-MCS-CMV-EGFP (GeneChem Co., Ltd.). The recombinational and packaging vectors pHelper 1.0 and 2.0 (GeneChem Co., Ltd.) were co-transfected into $293 \mathrm{~T}$ cells with Lipofectamine 2000 to produce viral particles of miR-34a antisense inhibitor and NC.

Animal model of $O A$. Experiments were performed according to the protocol approved by the Institutional Review Board of
Tangdu Hospital and the study was conducted in full compliance with the Declaration of Helsinki and Institutional Animal Care Standards. Twenty-eight 10-week-old male Sprague-Dawley rats (obtained from the Experimental Animal Center of Fourth Military Medical University) were used in the subsequent experiments. The rats were randomly divided into 4 groups $(n=7$ each): anti-miR-34a, non-specific NC, Sham and No Surgery. For the Sham group, identical surgical procedures were performed, although the ligaments and medial menisci were kept intact. The No Surgery group did not undergo an operation. The animals were anesthetized with 3\% pentobarbital sodium (Cat. no. 4579; Tocris, Bristol, UK). Briefly, a 10-mm medial parapatellar incision over the distal patella to proximal tibial plateau was made on the right knee joint of rat. Experimental OA was induced through anterior cruciate ligament transection and medial meniscus resection (ACLT+MMx) as previously described (21). The rats were allowed to move freely within the cages after surgery. Two weeks after surgery, the rats in the anti-miR-34a and NC groups received an intra-articular injection of $1 \times 10^{9}$ plaque forming units (PFU) of lentivirus vector encoding antisense miR-34a or non-specific control diluted in $100 \mu \mathrm{l}$ phosphate-buffered saline (PBS), respectively. However, the Sham and No Surgery groups received the same dose of normal saline.

Histological assessments. Ten weeks after surgery, the animals were sacrificed by cervical dislocation under $3 \%$ pentobarbital sodium narcosis and the whole joints were harvested. Tissues were routinely fixed in $10 \%$ buffered neutral formalin for $24 \mathrm{~h}$, decalcified in $10 \%$ ethylenediaminetetraacetic acid (EDTA) (pH 7.2) for 4 weeks, embedded into paraffin, and coronally sectioned at $6 \mu \mathrm{m}$. The sections were deparaffinized in xylene, hydrated with graded ethanol, and stained with Safranin O and Fast Green. Histological evaluation was performed according to the modified Mankin's score, as previously reported (22).

Statistical analysis. The continuous variables were presented as mean \pm standard deviation (SD). Statistical analysis was carried out using SPSS 20.0 (SPSS, Inc., Chicago, IL, USA). Comparisons between groups were made using the Student's t-test. One-way analysis of variance (ANOVA) followed by post hoc least significant difference (LSD) t-test was used for difference analysis in the multiple groups. Mankin's scores were evaluated using non-parametric statistical analyses. Results with values of $\mathrm{p}<0.05$ were considered significant.

\section{Results}

Expression of miR-34a and SIRT1 in healthy and OA human chondrocytes. To assess the potential involvement of miR-34a and SIRT1 in the OA process, we initially evaluated the expression levels between healthy and OA cartilage. As shown in Fig. 1A, miR-34a expression was significantly $(\mathrm{p}<0.01)$ increased in OA cartilage compared with healthy cartilage. Conversely, the expression level of SIRT1 in cartilage from OA patients was decreased $(\mathrm{p}<0.01)$ compared with healthy cartilage confirmed by quantitative PCR (Fig. 1B).

Confirmation of miR-34a oligonucleotide transfection into human chondrocytes. To investigate the function of miR-34a in OA, we transfected human chondrocytes with miR-34a 
A

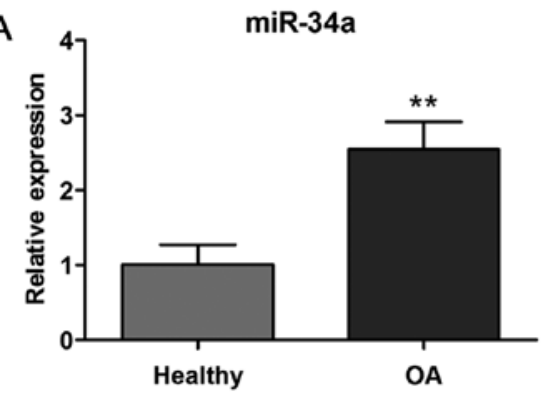

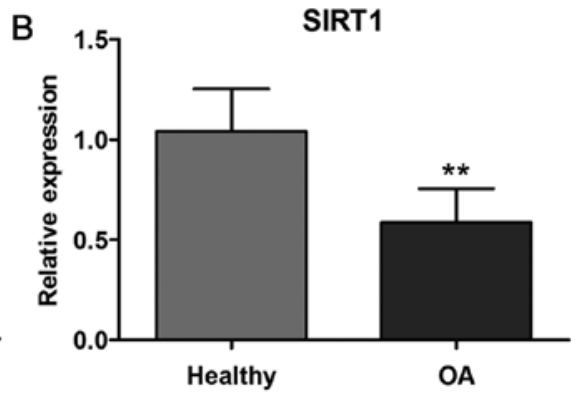

Figure 1. Expression of microRNA-34a (miR-34a) and silent information regulator 1 (SIRT1) in healthy and osteoarthritis (OA) cartilage. (A and B) Relative levels of miR-34a and SIRT1 mRNA in healthy $(\mathrm{n}=10)$ and OA $(\mathrm{n}=12)$ cartilage samples were determined by quantitative PCR, respectively. Small nuclear RNA U6 (RNU6) and GAPDH were used as endogenous controls. Data are shown as the means \pm SD. ${ }^{* *} \mathrm{P}<0.01$ compared to healthy samples.

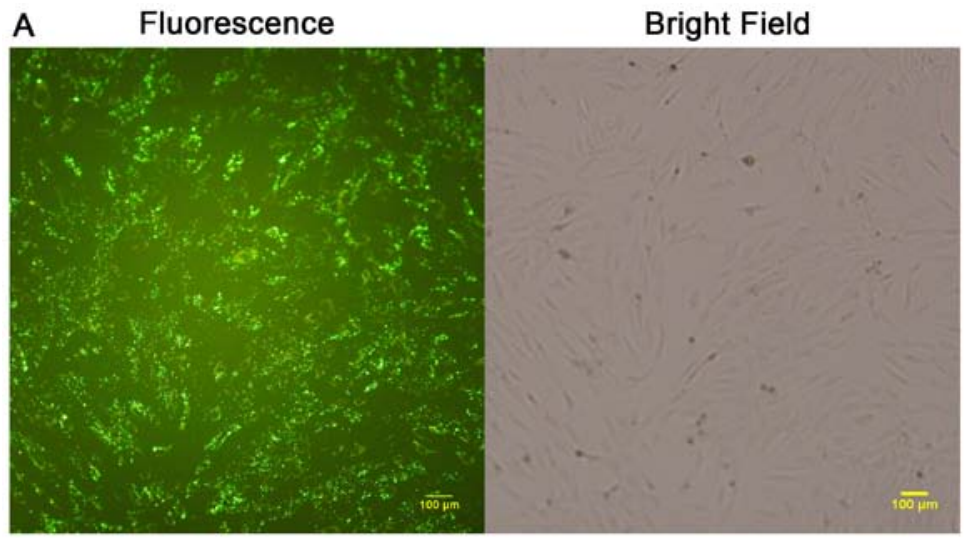

B

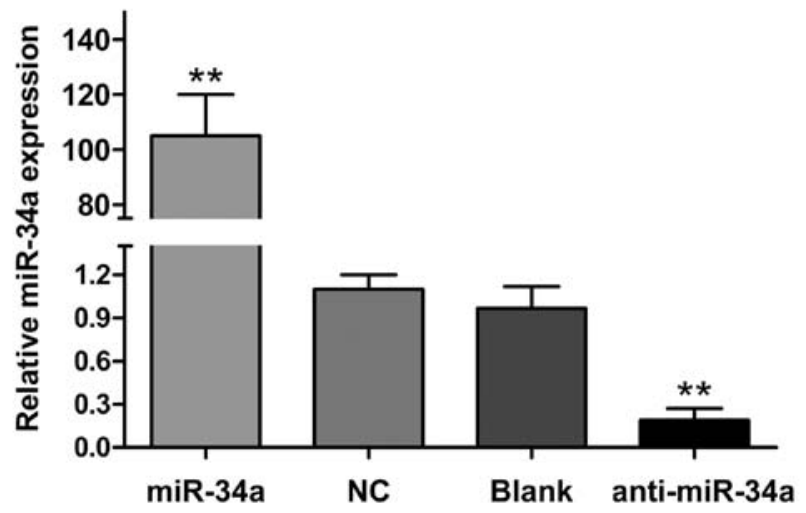

Figure 2. MicroRNA-34a (miR-34a) oligonucleotide transfection and miR-34a expression in human chondrocytes. Chondrocytes were transfected with miR-34a mimic (miR-34a), miR-34a inhibitor (anti-miR-34a) and negative oligonucleotide (NC). The blank cells were used as the control. (A) Fluorescence (left) and bright-field (right) images captured using fluorescence microscopy. (B) miR-34a expression levels in transfected chondrocytes. RNU6 was used as an internal control to normalize the results. Data are shown as the means \pm SD. ${ }^{* *} \mathrm{P}<0.01$ compared to respective controls.

mimic, miR-34a inhibitor and negative oligonucleotides, respectively. At $24 \mathrm{~h}$ after transfection, the fluorescence microscopy image showed that the transfection efficiency of miR-34a oligonucleotides into human chondrocytes reached $>80 \%$ (Fig. 2A). Compared with the NC and blank control (Blank) groups, the miR-34a level in the miR-34a group was elevated by $\sim 105$-fold $(\mathrm{p}<0.01)$. By contrast, the treatment of chondrocytes with miR-34a inhibitor decreased miR-34a expression by $80 \%(\mathrm{p}<0.01)$. No statistical significance of miR-34a expression was identified between the NC and Blank groups (Fig. 2B).
miR-34a inhibits the proliferation of chondrocytes in vitro. As miR-34a was markedly increased in OA chondrocyte, it may function as a promoter of OA. Therefore, the MTT assay was performed and proliferation curve was determined to examine the effects of miR-34a on the proliferation of human chondrocytes. Fig. 3 shows that chondrocytes transfected with miR-34a mimic exhibited a significant decrease in proliferation capacity compared with cells in the NC and Blank groups $(\mathrm{p}<0.01)$. By contrast, when transfected with the miR-34a inhibitor, chondrocytes grew at a higher rate $(\mathrm{p}<0.01)$. No statistical significance was observed in the proliferation rate between the $\mathrm{NC}$ and 


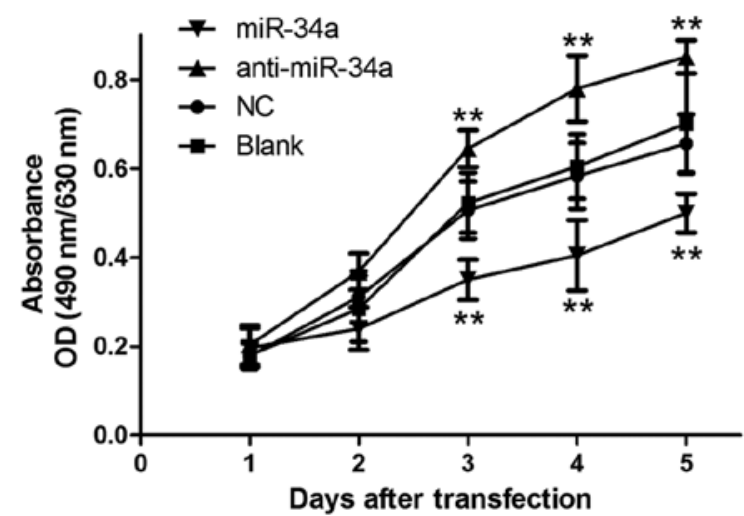

Figure 3. MicroRNA-34a (miR-34a) inhibits the proliferation of human chondrocytes in vitro. Chondrocytes were transfected with $100 \mathrm{nM}$ miR-34a mimic (miR-34a), miR-34a inhibitor (anti-miR-34a) and non-specific negative oligonucleotide. At 24, 48, 72, 96 or $120 \mathrm{~h}$ after transfection, cell proliferation was examined using the MTT assay. The cell number was evaluated as the absorbance at $490 \mathrm{~nm}$ with a reference wavelength of $630 \mathrm{~nm}$. Values of optica density (OD) are presented as the means $\pm \mathrm{SD}$. ${ }^{* *} \mathrm{P}<0.01$ compared to control.

Blank groups. The results indicated that overexpression of miR-34a inhibited the growth of human chondrocytes in vitro.

miR-34a regulates apoptosis of human chondrocytes in vitro. We performed Annexin V/PI staining-based flow cytometry analysis to evaluate the effects of miR-34a on chondrocyte apoptosis. Flow cytometry revealed that the chondrocyte apoptotic rate was increased significantly at $48 \mathrm{~h}$ after transfection with the miR-34a mimic $(\mathrm{p}<0.01)$. Cells in the anti-miR-34a group showed a lower proportion of Annexin V-positive cells $(\mathrm{p}<0.01)$. Cells transfected with non-specific oligonucleotides and blank cells had similar apoptotic rates (Fig. 4). Consequently, the results suggested that miR-34a regulates apoptosis of human chondrocytes in vitro.
miR-34a directly targets the SIRT1/p53 signaling pathway in human chondrocytes. miR-34a was found to directly repress the expression of SIRT1 in HCT116 cells (20). Our results in Fig. 1 show that cartilage from OA patients had a higher level of miR-34a and a lower level of SIRT1. Therefore, we further elucidated the molecular mechanism of miR-34a-mediated biological functions. The results from the western blot assay demonstrated that the SIRT1 protein level in the miR-34a group exhibited a significant decrease as compared with the $\mathrm{NC}$ and Blank groups. By contrast, knockdown of miR-34a by miR-34a inhibitor increased the protein abundance of SIRT1 (Fig. 5A and B). Additionally, the acetylated p53, a major target of SIRT1 deacetylation (16), was elevated by the ectopic expression of miR-34a and decreased by knockdown of miR-34a, while the total protein abundance of p53 was not significantly different between the groups (Fig. 5A, C and D). The protein levels of pro-apoptotic Bax and anti-apoptotic Bcl-2, two SIRT1/p53 pathway downstream genes, were elevated and inhibited, respectively, following miR-34a overexpression in human chondrocytes. The downregulation of miR-34a significantly decreased the Bax protein level and increased Bcl-2 (Fig. 5A, E and F). Furthermore, the levels of SIRT1 mRNA were not altered in chondrocytes transfected with miR-34a mimic or inhibitor (Fig. 5G).

To determine the underlying molecular mechanisms of miR-34a-mediated regulation of SIRT1 expression in human chondrocytes, we developed luciferase reporter vectors containing the wild-type 3'-UTR or mutant-type 3'-UTR of miR-34a binding sites of SIRT1 and detected the effects of miR-34a on the luciferase activity in human chondrocytes. Luciferase analysis revealed that the transfection of miR-34a mimic significantly suppressed the luciferase activity of the wild-type reporter vector while mutation of the miR-34a binding sites blocked this suppressive effect $(\mathrm{p}<0.05)($ Fig. $5 \mathrm{H})$. These data suggest that miR-34a affects SIRT1 expression by
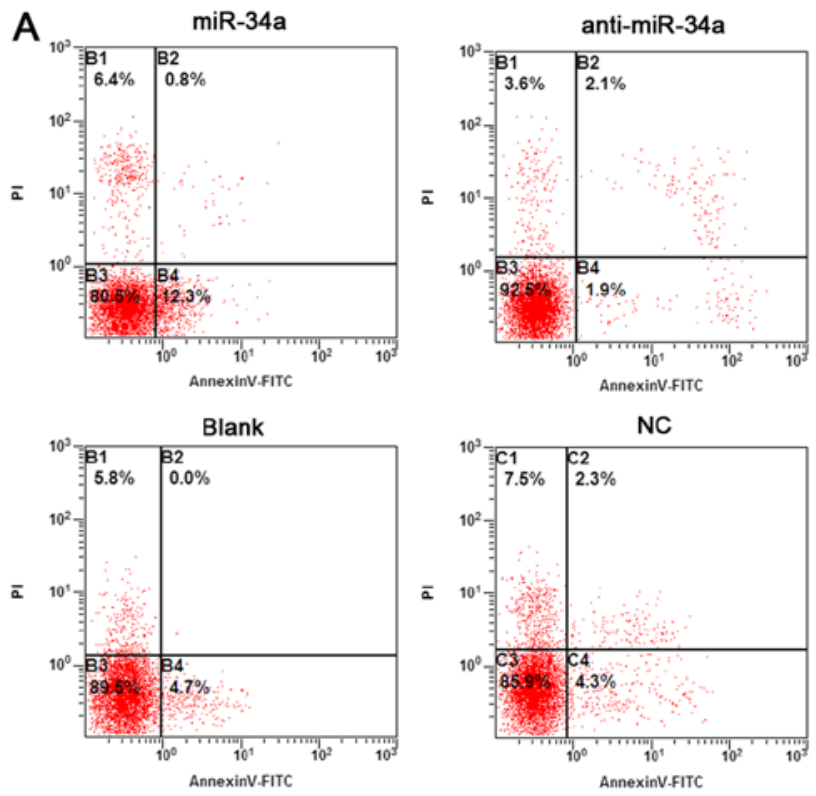

B

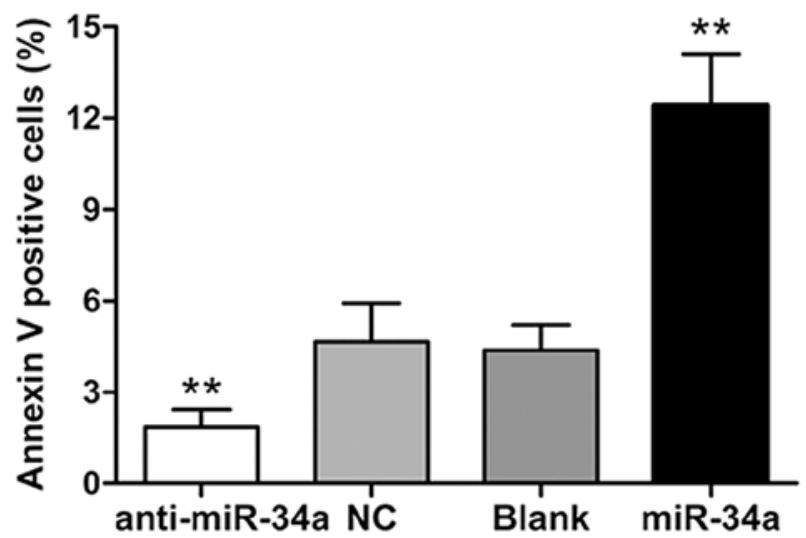

Figure 4. MicroRNA-34a (miR-34a) regulates the apoptosis of human chondrocytes in vitro. Chondrocytes were transfected with 100 nM miR-34a mimic (miR-34a), miR-34a inhibitor (anti-miR-34a) and non-specific negative oligonucleotide (NC). The blank cells were set as the control. Forty-eight hours after transfection, Annexin V/PI staining-based flow cytometry was performed to evaluate apoptosis of the four groups. (A and B) Apoptosis of transfected chondrocytes. Data are shown as the means $\pm \mathrm{SD} .{ }^{* *} \mathrm{P}<0.01$ compared to respective controls. 


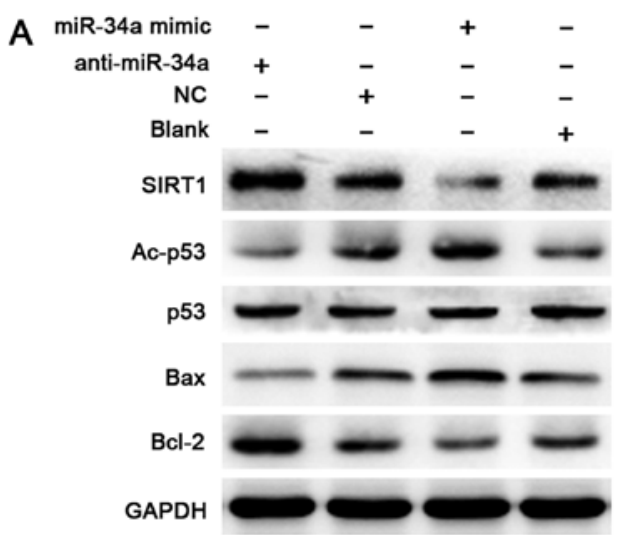

\section{B}

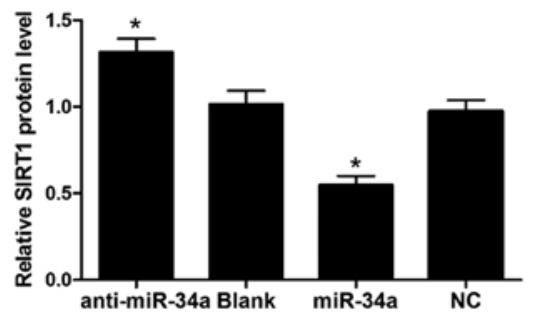

C

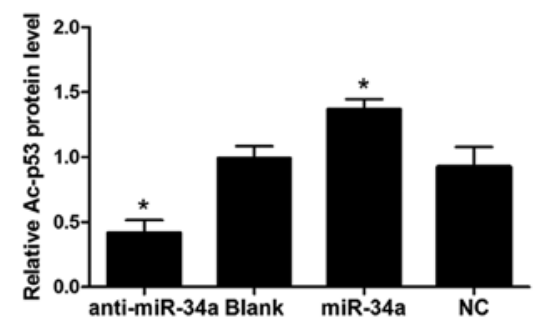

E

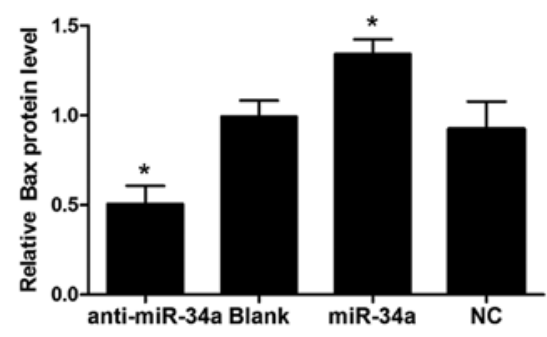

G

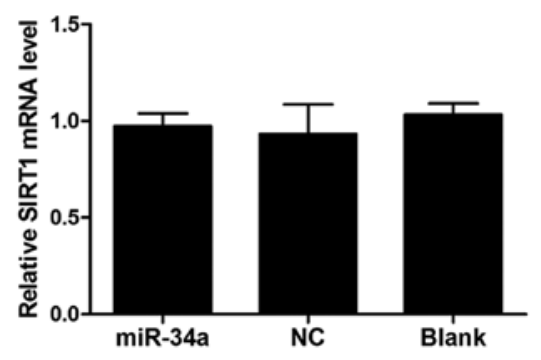

D
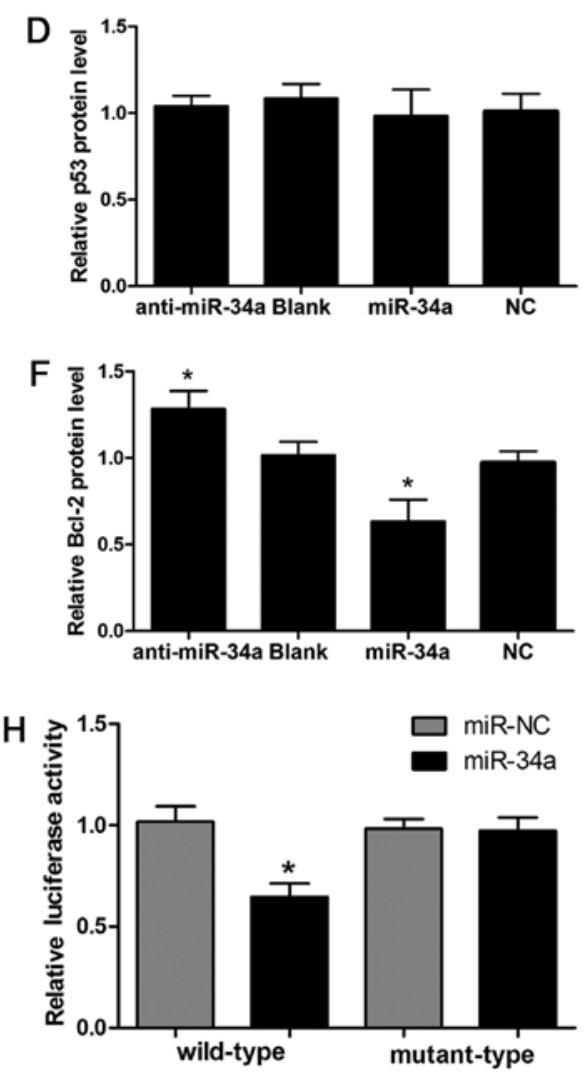

Figure 5. MicroRNA-34a (miR-34a) directly targets silent information regulator 1 (SIRT1)/p53 signaling pathway in human chondrocytes. (A-F) Western blot analysis of SIRT1, acetylated p53 (ac-p53), p53, Bax, Bcl-2 protein expression. GAPDH was used as a loading control. (G) mRNA levels of SIRT1 in miR34a, negative control (NC), Blank group normalized to GAPDH. (H) The luciferase reporter assay for the 3'-untranslated region (3'-UTR) of SIRT1 mRNA. Chondrocytes were co-transfected with wild-type SIRT1 3'-UTR reporter plasmid or mutant-type SIRT1 3'-UTR reporter plasmid plus miR-34a mimic (miR-34a) or non-specific negative oligonucleotide (miR-NC). At $48 \mathrm{~h}$ after transfection, luciferase activities were measured. Data are shown as the means $\pm \mathrm{SD}$. "P $<0.05$ compared with blank and NC.

post-transcriptional regulation and miR-34a directly targets the SIRT1/p53 signaling pathway in human chondrocytes.

Suppression of miR-34a ameliorates the surgery-induced progression of $O A$ in rats. Given the findings in vitro, animal studies were conducted to evaluate the effects of miR-34a on experimental OA. In vivo, lentiviruses encoding miR-34a antisense inhibitor or NC were injected into the knee joints of rats. Cartilage was harvested for histological evaluation. The results revealed cartilage destruction and decreased Safranin O staining in the NC group. Intra-articular injection of miR-34a inhibitor-expressing lentiviruses significantly attenuated surgery-induced cartilage destruction in the anti-miR-34a group (Fig. 6A). Fig. 6B shows the modified Mankin's score in the miR-34a inhibitor-treated joints was significantly lower than that for NC-treated joints $(\mathrm{p}<0.01)$.

\section{Discussion}

OA is a degenerative joint disorder with multifactorial etiology caused by risk factors such as ageing, obesity and trauma (23). Over the past few years, microRNAs have received attention for their essential roles in cartilage homeostasis. However, the ectopic expression of microRNAs occurs during OA progression. It has been reported that modulation of miR-145 affects the expression of Smad3 causing a change of its downstream target 

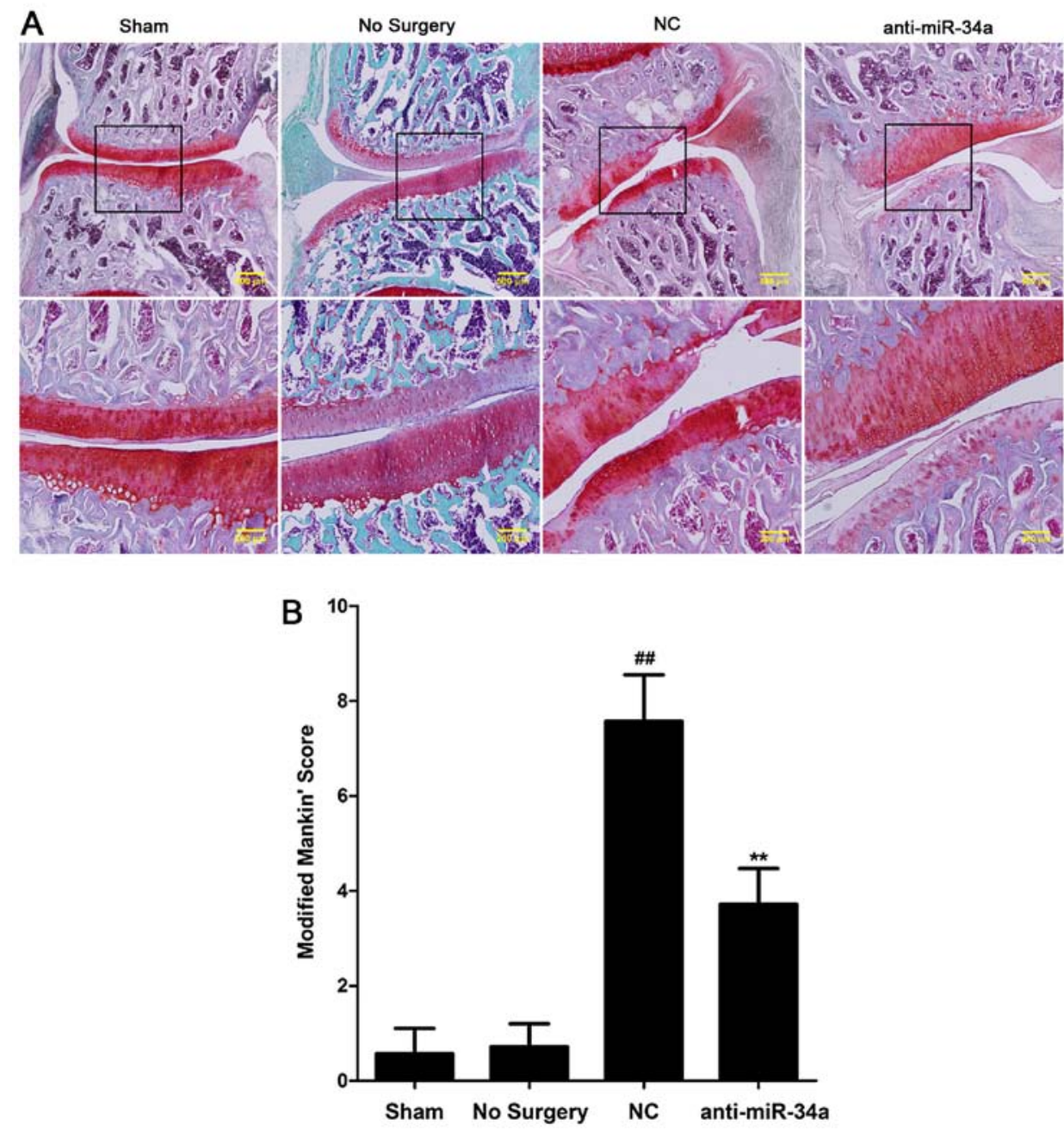

Figure 6. Suppression of microRNA-34a (miR-34a) ameliorates surgery-induced cartilage degradation. (A) Histological observation of sections stained with Safranin O and counterstained with fast green 8 weeks after injection. Bar, $500 \mu \mathrm{m}$ (magnification, $\mathrm{x} 40$ ) and $200 \mu \mathrm{m}$ (magnification, x100). (B) Mankin's scores for each group. Data are presented as the means $\pm \mathrm{SD} .{ }^{* *} \mathrm{P}<0.01$ compared with the $\mathrm{NC}$ group, and ${ }^{\# \#} \mathrm{p}<0.01$ compared with the Sham and No Surgery groups.

gene expression as well as IL-1 $\beta$-induced ECM degradation in OA chondrocytes (24). Jin et al have demonstrated that miR-146a is involved in human chondrocyte apoptosis in response to mechanical injury and contribute to the pathogenesis of OA by modulating the VEGF and TGF- $\beta$ signaling pathway (25). In a previous study, silencing of miR-34a effectively reduced rat chondrocyte apoptosis caused by IL-1 $\beta$ (18). However, the molecular mechanism and the role of miR-34a in human chondrocyte as well as OA progression remain to be investigated.

Members of the miR-34 family are direct transcriptional targets of p53 while the ectopic expression of miR-34 induces apoptosis, cell cycle arrest, senescence and other biological behavior $(14,26,27)$. In the present study, we observed that the expression of miR-34a was significantly increased in primary chondrocytes from OA patients compared with healthy chondrocytes from traumatic amputees, which indicates involvement of miR-34a in the pathogenesis of OA. To determine the function of miR-34a in OA, we used chemically synthesized oligonucleotides to manipulate the expression of miR-34a. The results showed that these oligonucleotides were efficiently transfected into human chondrocyte in vitro and significantly increased or decreased miR-34a expression levels, facilitating the study of miR-34a function.
Mounting evidence shows that chondrocyte apoptosis plays a crucial role in the mechanisms of degeneration and degradation of articular cartilage in OA $(6,7)$. Thus, the mechanism of apoptosis offers potentially useful therapeutic targets for the management of this chronic disease (28). In the present study, results from the flow cytometric analysis and MTT assay revealed that transfection of a synthetic miR-34a mimic in vitro significantly promoted apoptosis and inhibited the proliferation in human chondrocytes, whereas the downregulation of miR-34a led to significant suppression of apoptosis and enhanced cell viability.

SIRT1, a member of sirtuin family, functions as a histone deacetylase and has been linked with age-associated diseases such as diabetes type II, Alzheimer's and osteoporosis $(29,30)$. Increasing evidence suggests that SIRT1 has a key role in OA. Inhibition of SIRT1 promoted the development of OA by suppressing aggrecan expression and increasing the levels of COL10A1 and ADAMTS-5 in human chondrocytes (31). Inactivation of SIRT1 promotes apoptosis through mitochondria-related signals in chondrocytes (32). Enhanced acetylation of the well-known tumor suppressor gene $p 53$ in response to types of cell stress is crucial for p53-mediated apoptosis, cell growth arrest and transcriptional 
activities $(33,34)$. Thus, SIRT1 may regulate p53 function by deacetylating p53. Chung et al reported that the overexpression of SIRT1 significantly protects fibroblasts from UVB-induced cellular senescence by suppressing UVB-induced p53 acetylation and its transcriptional activity (35).

Notably, it has been reported that repression of SIRT1 by miR-34a regulates apoptosis in colon cancer cells (20). Thus, miR-34a induces apoptosis in human chondrocyte by modulating SIRT1/p53 signaling during the pathogenesis of OA. In the present study, we observed a significantly decreased expression of SIRT1 in OA chondrocytes, consistent with previous studies (32), showing the involvement of SIRT1 in OA. We also found an interaction between miR-34a and SIRT1/p53 signaling. Western blot analysis revealed that the SIRT1 protein level was considerably decreased in miR-34a-mimic-transfected cells, leading to an increase of acetylated p53, Bax and decrease of Bcl-2. Additionally, results from the luciferase reporter assay demonstrated that miR-34a suppressed the luciferase activity of the wild-type SIRT1 3'-UTR vector, while mutation of the miR-34a binding site attenuated this suppressive effect, indicating that miR-34a inhibits SIRT1 by directly binding to the 3'-UTR of SIRT1 mRNA.

A classical ACLT+MMx rat model is considered an appropriate platform to verify the biological effects of miR-34a in OA in vivo, which is feasible and reproducible. Lentivirus-mediated gene delivery has several advantages, including efficient transduction into a wide variety of dividing or non-dividing cells and stable expression of transgenes (36), and has been applied to studies on arthritis in vivo $(37,38)$. In the current study, histological findings showed that articular cartilage damage and severity of disease could be attenuated by the intra-articular injection of lentivirus encoding miR-34a antisense inhibitor. To the best of our knowledge, the present study provides the first evidence that the downregulation of miR-34a mediated by intra-articular injection of lentivirus ameliorates OA progression in rat induced by surgery. Progressive degeneration of articular cartilage has been considered the main event underlying the pathogenesis of OA, leading to loss of physiological function $(5,39)$. However, current drug therapy of OA remains unsatisfactory and may not reverse the destruction of the articular cartilage while end-stage patients have to resort to joint replacement surgery $(40,41)$. Thus, the administration of therapeutic agents that potentially prevent degradation, inhibit inflammation or promote cartilage self-repairing is a promising therapeutic approach.

Taken together, we suggest that miR-34a promotes apoptosis and inhibits proliferation by directly regulating the SIRT1/p53 signaling pathway in primary human chondrocytes. Silencing miR-34a by intra-articular injection of lentivirus may attenuate disease progression in a rat model of OA. Additional studies examining the clinical potential of miR-34a and its target SIRT1 in OA treatment are required. Detailed investigation into the network of mediators involved in miR-34a regulation may accelerate the development of a novel therapeutic approach in OA.

\section{Acknowledgements}

The present study was funded by grants from the National Natural Science Foundation of China (nos. 81072194 and 81201633 ). The funders had no role in the study design.

\section{References}

1. Lawrence RC, Helmick CG, Arnett FC, Deyo RA, Felson DT, Giannini EH, Heyse SP, Hirsch R, Hochberg MC, Hunder GG, et al: Estimates of the prevalence of arthritis and selected musculoskeletal disorders in the United States. Arthritis Rheum 41: 778-799, 1998.

2. Gore M, Tai KS, Sadosky A, Leslie D and Stacey BR: Clinical comorbidities, treatment patterns, and direct medical costs of patients with osteoarthritis in usual care: A retrospective claims database analysis. J Med Econ 14: 497-507, 2011.

3. Duncan R, Peat G, Thomas E, Hay E, McCall I and Croft P: Symptoms and radiographic osteoarthritis: not as discordant as they are made out to be? Ann Rheum Dis 66: 86-91, 2007.

4. Findlay DM and Atkins GJ: Osteoblast-chondrocyte interactions in osteoarthritis. Curr Osteoporos Rep 12: 127-134, 2014.

5. Goldring MB: The role of the chondrocyte in osteoarthritis. Arthritis Rheum 43: 1916-1926, 2000.

6. Blanco FJ, Guitian R, Vázquez-Martul E, de Toro FJ and Galdo F: Osteoarthritis chondrocytes die by apoptosis. A possible pathway for osteoarthritis pathology. Arthritis Rheum 41: 284-289, 1998.

7. Hashimoto S, Ochs RL, Komiya S and Lotz M: Linkage of chondrocyte apoptosis and cartilage degradation in human osteoarthritis. Arthritis Rheum 41: 1632-1638, 1998.

8. Bartel DP: MicroRNAs: Genomics, biogenesis, mechanism, and function. Cell 116: 281-297, 2004.

9. Ambros V: The functions of animal microRNAs. Nature 431: 350-355, 2004.

10. Farh KK, Grimson A, Jan C, Lewis BP, Johnston WK, Lim LP, Burge CB and Bartel DP: The widespread impact of mammalian MicroRNAs on mRNA repression and evolution. Science 310: 1817-1821, 2005.

11. Miyaki S and Asahara H: Macro view of microRNA function in osteoarthritis. Nat Rev Rheumatol 8: 543-552, 2012.

12. Goldring MB and Marcu KB: Epigenomic and microRNA-mediated regulation in cartilage development, homeostasis, and osteoarthritis. Trends Mol Med 18: 109-118, 2012.

13. Tazawa $\mathrm{H}$, Tsuchiya $\mathrm{N}$, Izumiya $\mathrm{M}$ and Nakagama $\mathrm{H}$ : Tumor-suppressive miR-34a induces senescence-like growth arrest through modulation of the E2F pathway in human colon cancer cells. Proc Natl Acad Sci USA 104: 15472-15477, 2007.

14. Chang TC, Wentzel EA, Kent OA, Ramachandran K, Mullendore M, Lee KH, Feldmann G, Yamakuchi M, Ferlito M, Lowenstein CJ, et al: Transactivation of miR-34a by p53 broadly influences gene expression and promotes apoptosis. Mol Cell 26: 745-752, 2007.

15. Luo J, Nikolaev AY, Imai S, Chen D, Su F, Shiloh A, Guarente L and Gu W: Negative control of p53 by Sir2alpha promotes cell survival under stress. Cell 107: 137-148, 2001.

16. Vaziri H, Dessain SK, Ng Eaton E, Imai SI, Frye RA, Pandita TK, Guarente L and Weinberg RA: hSIR2(SIRT1) functions as an NAD-dependent p53 deacetylase. Cell 107: 149-159, 2001.

17. Kobayashi Y, Furukawa-Hibi Y, Chen C, Horio Y, Isobe K, Ikeda K and Motoyama N: SIRT1 is critical regulator of FOXO-mediated transcription in response to oxidative stress. Int J Mol Med 16: 237-243, 2005.

18. Abouheif MM, Nakasa T, Shibuya H, Niimoto $T$, Kongcharoensombat W and Ochi M: Silencing microRNA-34a inhibits chondrocyte apoptosis in a rat osteoarthritis model in vitro. Rheumatology (Oxford) 49: 2054-2060, 2010.

19. Matsushita T, Sasaki H, Takayama K, Ishida K, Matsumoto T, Kubo S, Matsuzaki T, Nishida K, Kurosaka M and Kuroda R: The overexpression of SIRT1 inhibited osteoarthritic gene expression changes induced by interleukin- $1 \beta$ in human chondrocytes. J Orthop Res 31: 531-537, 2013.

20. Yamakuchi M, Ferlito M and Lowenstein CJ: miR-34a repression of SIRT1 regulates apoptosis. Proc Natl Acad Sci USA 105: 13421-13426, 2008.

21. Hayami T, Pickarski M, Zhuo Y, Wesolowski GA, Rodan GA and Duong LT: Characterization of articular cartilage and subchondral bone changes in the rat anterior cruciate ligament transection and meniscectomized models of osteoarthritis. Bone 38: 234-243, 2006.

22. Mankin HJ, Dorfman H, Lippiello L and Zarins A: Biochemical and metabolic abnormalities in articular cartilage from osteo-arthritic human hips. II. Correlation of morphology with biochemical and metabolic data. J Bone Joint Surg Am 53: 523-537, 1971. 
23. Pottie P, Presle N, Terlain B, Netter P, Mainard D and Berenbaum F: Obesity and osteoarthritis: More complex than predicted! Ann Rheum Dis 65: 1403-1405, 2006

24. Yang B, Kang X, Xing Y, Dou C, Kang F, Li J, Quan Y and Dong S: Effect of microRNA-145 on IL-1 $\beta$-induced cartilage degradation in human chondrocytes. FEBS Lett 588: 2344-2352, 2014.

25. Jin L, Zhao J, Jing W, Yan S, Wang X, Xiao C and Ma B: Role of miR-146a in human chondrocyte apoptosis in response to mechanical pressure injury in vitro. Int J Mol Med 34: 451-463, 2014.

26. He L, He X, Lim LP, de Stanchina E, Xuan Z, Liang Y, Xue W, Zender L, Magnus J, Ridzon D, et al: A microRNA component of the p53 tumour suppressor network. Nature 447: 1130-1134, 2007.

27. Raver-Shapira N, Marciano E, Meiri E, Spector Y, Rosenfeld N, Moskovits N, Bentwich Z and Oren M: Transcriptional activation of miR-34a contributes to p53-mediated apoptosis. Mol Cell 26: 731-743, 2007

28. Johnson EO, Charchandi A, Babis GC and Soucacos PN Apoptosis in osteoarthritis: morphology, mechanisms, and potential means for therapeutic intervention. J Surg Orthop Adv 17: 147-152, 2008.

29. Michan S and Sinclair D: Sirtuins in mammals: insights into their biological function. Biochem J 404: 1-13, 2007

30. Zeng L, Chen R, Liang F, Tsuchiya H, Murai H, Nakahashi T, Iwai K, Takahashi T, Kanda T and Morimoto S: Silent information regulator, Sirtuin 1, and age-related diseases. Geriatr Gerontol Int 9: 7-15, 2009.

31. Fujita N, Matsushita T, Ishida K, Kubo S, Matsumoto T, Takayama K, Kurosaka M and Kuroda R: Potential involvement of SIRT1 in the pathogenesis of osteoarthritis through the modulation of chondrocyte gene expressions. J Orthop Res 29: 511-515, 2011.

32. Takayama K, Ishida K, Matsushita T, Fujita N, Hayashi S, Sasaki K, Tei K, Kubo S, Matsumoto T, Fujioka H, et al: SIRT1 regulation of apoptosis of human chondrocytes. Arthritis Rheum 60: 2731-2740, 2009.
33. Knights CD, Catania J, Di Giovanni S, Muratoglu S, Perez R, Swartzbeck A, Quong AA, Zhang X, Beerman T, Pestell RG, et al: Distinct p53 acetylation cassettes differentially influence gene-expression patterns and cell fate. J Cell Biol 173: 533-544, 2006.

34. Sykes SM, Mellert HS, Holbert MA, Li K, Marmorstein R, Lane WS and McMahon SB: Acetylation of the p53 DNA-binding domain regulates apoptosis induction. Mol Cell 24: 841-851, 2006.

35. Chung KW, Choi YJ, Park MH, Jang EJ, Kim DH, Park BH, Yu BP and Chung HY: Molecular insights into SIRT1 protection against UVB-induced skin fibroblast senescence by suppression of oxidative stress and p53 acetylation. J Gerontol A Biol Sci Med Sci 70: 959-968, 2015.

36. Singer O and Verma IM: Applications of lentiviral vectors for shRNA delivery and transgenesis. Curr Gene Ther 8: 483-488, 2008.

37. Chen SY, Shiau AL, Li YT, Lin YS, Lee CH, Wu CL and Wang CR: Suppression of collagen-induced arthritis by intra-articular lentiviral vector-mediated delivery of Toll-like receptor 7 short hairpin RNA gene. Gene Ther 19: 752-760, 2012.

38. Shen PC, Lu CS, Shiau AL, Lee CH, Jou IM and Hsieh JL: Lentiviral small hairpin RNA knockdown of macrophage inflammatory protein- $1 \gamma$ ameliorates experimentally induced osteoarthritis in mice. Hum Gene Ther 24: 871-882, 2013.

39. Aigner T, Haag J, Martin J and Buckwalter J: Osteoarthritis: aging of matrix and cells - going for a remedy. Curr Drug Targets 8: 325-331, 2007

40. Vangsness CT Jr, Spiker W and Erickson J: A review of evidence-based medicine for glucosamine and chondroitin sulfate use in knee osteoarthritis. Arthroscopy 25: 86-94, 2009.

41. Liu XW, Zi Y, Xiang LB and Wang Y: Total hip arthroplasty: a review of advances, advantages and limitations. Int J Clin Exp Med 8: 27-36, 2015. 